\title{
Estudio de la relación entre estrategias de aprendizaje y desempeño académico en estudiantes de medicina
}

\author{
Study of the relationship between learning strategies and academic performance \\ in medical students
}

Mildred López'1 Yolanda Heredia Escorza²

\section{Resumen}

Introducción: las estrategias de aprendizaje utilizadas por los estudiantes de medicina han evolucionado con el paso del tiempo, avanzando de modelos más tradicionales hacia la autodirección y autonomía del aprendizaje, propios de un aprendiz de por vida. El objetivo de este estudio fue evaluar la relación entre estas estrategias de aprendizaje y el desempeño académico de estudiantes de medicina. Materiales y métodos: el enfoque y diseño de la investigación es cuantitativo y correlacional. La muestra consistió en 144 estudiantes de una universidad privada del norte de México. Como instrumento se utilizó el cuestionario para la evaluación de las estrategias de aprendizaje de los estudiantes universitarios, y una pregunta donde los estudiantes reportaban su desempeño académico con el promedio acumulado. El análisis de datos considera el uso de estadística descriptiva a fin de explorar el desempeño académico y estrategia, y correlacional para entender la vinculación entre escalas. Como factor de interés para el análisis se integraron también las variables de género, edad, y grado de escolaridad del padre y madre. Resultados: los estudiantes muestran una media y desviación estándar (DE) de 9,22 $\pm 0,41$ en el desempeño académico (escala del 1 al 10). Las estrategias de aprendizaje con medias más altas fueron motivación intrínseca con una media de 4,69 $\pm 0,56$ y valor de la tarea con una media de 4,67 $\pm 0,54$. Las tendencias menos favorables fueron en atribuciones externas con media de 2,55 $\pm 0,78$ y repetición simple con una media de 2,82 1,12. La correlación entre las variables de desempeño académico y las subescalas de estrategias es débil, varían entre -0,01 con la subescala de componentes afectivos y 0,16 con la subescala de estrategias de control del contexto, interacción social y manejo de recursos. Discusión: los resultados del desempeño académico reportados por los estudiantes son congruentes con los altos parámetros de selección del programa de medicina. La falta de relación entre esta variable y las estrategias de aprendizaje contradicen la creencia de que la selección y activación de estas estrategias determina el desempeño. Es importante que al interior de los programas se estudien las estrategias utilizadas por los estudiantes, a fin de facilitar recursos para movilizarlos y potenciar su aprendizaje.

Palabras clave: estrategias de aprendizaje; desempeño académico; estudiantes de medicina.

\begin{abstract}
Introduction: The learning strategies used by medical students have evolved, moving from more traditional models to self-direction and autonomy of learning, part of life-long-learners. The objective of this study was to assess the relationship between learning strategies and the academic performance of medical students. Methods: The focus and design of the research are quantitative, correlational, and cross-sectional. The sample consisted of 144 students at a private university in northern Mexico. As an instrument, we used the Questionnaire to assess university students' learning strategies and a self-report of grades average on the program. The analysis of data analysis considers descriptive statistics to explore academic performance and each strategy and correlational to understand the link between the scales. As a factor of analysis, the variables of gender, age, and degree of schooling of the father and mother were used. Results: The results show a mean and standard deviation (SD) of 9.22 $\pm 0,41$ in academic performance (scale of 1 to 10). The strategies with the highest mean were intrinsic motivation with a mean of $4.69 \pm 0.56$, and task valuation with a mean of $4.67 \pm 0.54$. The lowest score were found on external attribution with a mean of $2.55 \pm 0.78$ and simple repetition with a mean of $2.82 \pm 1.12$. The analysis indicates that the correlation between academic performance and the strategies of the different subscales is weak, varying between - 0.01 with the subscale of affective components and 0.16 with the subscale of context control strategies, social interaction, and resource management.
\end{abstract}

(1) Tecnológico de Monterrey, Escuela de Medicina y Ciencias de la Salud Monterrey, Nuevo León, México.

(2) Tecnológico de Monterrey, Nuevo León, México.

Autor de correspondencia: mildredlopez@tec.mx 
Discussion: The results of the academic performance reported by students are congruent with the parameters for selection in the medical program. The lack of correlation between this variable and the strategies contradicts the belief that selection and activation of these strategies dictate performance. Educational programs must study the strategies used by participants to facilitate resources to mobilize and activate their learning.

Keywords: learning strategies; academic performance; medical students.

Fecha de envío: 2021-05-26 - Fecha de aceptación: 2021-06-23

\section{Introducción}

Cuando los estudiantes ingresan a un programa de educación superior enfrentan una transición importante, por un lado, deben acoplarse a un modelo educativo más autodirigido que el bachillerato o preparatoria, en el que deben diseñar sus propias estrategias de aprendizaje (Biwer et al., 2020). En un segundo nivel, emprenden un viaje de descubrimiento de su identidad profesional en el que se convierten en médicos, psicólogos, nutriólogos, odontólogos, kinesiólogos etc. (Quiroga-Garza et al., 2020). Naturalmente, recurren a la estrategia de releer sus notas del contenido revisado en clase o memorizar mediante repetición, y aunque esto pudiera ayudarles a aprobar un examen, estas estrategias son poco efectivas en el largo plazo.

Tradicionalmente, ha existido la creencia de que existe una relación entre estas estrategias de aprendizaje y el desempeño académico (Gargallo et al., 2009; Salazar \& Heredia, 2019). Diversas perspectivas teóricas, así como investigaciones recientes, han tratado de vincular los componentes cognitivos y las regulaciones de hábitos y motivaciones, detrás del proceso enseñanza-aprendizaje (Rinaudo et al., 2003; Pérez \& La Cruz, 2014). Obergriesser \& Stoeger (2020) se enfocaron a analizar precepciones del logro, así como la satisfacción o el aburrimiento que los estudiantes reportan en sus estudios, para poder predecir su desempeño académico. Para Hayat et al. (2020) la confianza en la selección y aplicación de las estrategias de aprendizaje se traduce en autoeficacia para completar tareas académicas y adquirir los saberes descritos en los objetivos de aprendizaje.

Las estrategias de aprendizaje son planes de acción para facilitar la adquisición y aplicación del conocimiento (Pérez \& La Cruz, 2014). Estos planes se desarrollan de manera interna en cada individuo (Schunk, 1997; Navea, 2018), y activan el proceso de "aprender a aprender" (Páez, 2006; Valdez García et al., 2017). Marzano \& Kendall (2007) lo describen en tres sistemas: el interno que se conforma por una serie de creencias cuando el sujeto considera involucrarse en una tarea. El segundo sistema, el metacognitivo, propone metas y genera estrategias que le permitan alcanzar las metas. El tercero, el cognitivo, es el encargado de las operaciones mentales como: comparaciones, inferencias, análisis, clasificaciones, etc.
Las estrategias también alcanzan distintos niveles de profundidad (Pintrich et al., 1991). El nivel más bajo se refiere a la repetición de la información. En un segundo nivel, se modifica e interpreta la información en sus propias palabras. En un tercer nivel, se integra la información nueva a las estructuras ya existentes, establece conexiones significativas otorgando sentido y coherencia al material. En un cuarto nivel, surgen los procesos de reflexión y cuestionamiento del material de trabajo (Pintricht \& García, 1993; Gagné, 1996).

El desempeño académico se puede definir como un indicador que permite conocer el nivel de conocimientos y destrezas expresadas por los estudiantes durante el proceso de evaluación. Edel (2003), lo define como un constructo a través del cual existe una aproximación a la evidencia y dimensión del perfil de habilidades, conocimientos, actitudes y valores desarrollados por el alumno en el proceso de enseñanza-aprendizaje (Garbanzo, 2007; Ramos \& Pacheco, 2017).

Las variables personales proporcionan información de tipo tanto cognitivo como emocional que son inherentes a la persona. Los factores como el nivel socioeconómico y cultural de los padres, el apoyo para la escolarización y los institucionales que tienen que ver con la organización escolar, las credenciales de los profesores, la práctica docente y de evaluación.

De tal forma que el objetivo principal de la presente investigación fue evaluar la relación entre las estrategias de aprendizaje y el desempeño académico de los estudiantes. Como objetivos secundarios de este estudio se encuentra el determinar qué estrategias afectivas, de apoyo y control activan los estudiantes de la carrera de medicina. Así como determinar qué estrategias relacionadas con el procesamiento de la información activan los estudiantes de la carrera de medicina

\section{Materiales y método}

El enfoque y diseño de la investigación es cuantitativo, correlacional y transversal, al buscar explicar mediante un abordaje positivista la vinculación entre distintas escalas que conforman el instrumento. Como investigación correlacional pretende responder a preguntas de investigación mediante la asociación de variables con un patrón predecible para una población (Hernández et al., 2010). 
La muestra constó de 144 estudiantes de pregrado de medicina de una universidad privada del norte de México que contestaron el instrumento. El estudio se llevó a cabo según la Declaración de Helsinki, y los lineamientos del Comité Institucional de Ética e Investigación institucional que aprobó el protocolo. Todos los participantes participaron voluntariamente en la experiencia, y todos ellos aceptaron ser incluidos en el estudio; su elección quedó documentada en un consentimiento informado por escrito.
Como instrumento se utilizó el cuestionario para la evaluación de las estrategias de aprendizaje de los estudiantes universitarios (CEVEAPEU) que, en su versión original, cuenta con 88 ítems agrupados en dos factores: estrategias afectivas, de apoyo y control, y la de estrategias relacionadas con el procesamiento de la información (Gargallo et al., 2009). Sus seis sub-escalas se describen en la tabla 1.

Tabla 1: Descripción de las sub-escalas del instrumento.

\begin{tabular}{|c|c|c|}
\hline Escala & Subescala & Descripción \\
\hline \multirow{4}{*}{$\begin{array}{l}\text { Estrategias afectivas, } \\
\text { de apoyo y } \\
\text { control }\end{array}$} & Estrategias motivacionales. & $\begin{array}{l}\text { Se refiere a como el aprendizaje se ve activado por la motivación, reconocer } \\
\text { el valor de la tarea y persistir para alcanzarla. Además, integra la perspectiva } \\
\text { de autoeficacia y las expectativas sobre el éxito futuro. }\end{array}$ \\
\hline & Componentes afectivos. & $\begin{array}{l}\text { Refiere la influencia en el aprendizaje del estado anímico, como la ansie- } \\
\text { dad, y el físico. }\end{array}$ \\
\hline & Estrategias metacognitivas. & $\begin{array}{l}\text { Se refiere a cómo el conocimiento sobre estrategias, y la elección de estra- } \\
\text { tegias para la planeación, control y evaluación impactan en el aprendizaje. }\end{array}$ \\
\hline & $\begin{array}{l}\text { Estrategias de control del contexto, interacción } \\
\text { social y manejo de recursos. }\end{array}$ & $\begin{array}{l}\text { Refiere cómo el contexto social alrededor del estudiante tiene una influencia } \\
\text { en el aprendizaje. Por ejemplo, en la interacción con sus compañeros, } \\
\text { profesores, y otras comunidades de aprendizaje. }\end{array}$ \\
\hline \multirow{2}{*}{$\begin{array}{l}\text { Estrategias relacio- } \\
\text { nadas con el proce- } \\
\text { samiento } \\
\text { de la información }\end{array}$} & Estrategia de búsqueda y selección de información. & $\begin{array}{l}\text { Refiere los recursos de la persona para buscar y utilizar fuentes confia- } \\
\text { bles de información, así como discriminar entre esta para identificar la } \\
\text { confiabilidad de estas. }\end{array}$ \\
\hline & Estrategias de procesamiento y uso de la información. & $\begin{array}{l}\text { Se refiere al uso de recursos para organizar información, personalizarla } \\
\text { de forma creativa, y establecer caminos para su análisis y recuperación. }\end{array}$ \\
\hline
\end{tabular}

Además, se solicitó a los participantes que reportaran su desempeño académico con el promedio acumulado en el pregrado.

El análisis de los datos considera el uso de estadística descriptiva a fin de explorar el desempeño académico y las tendencias en cada estrategia, y correlacional para entender la vinculación entre las distintas escalas. La primera parte estudió la media y desviación estándar del desempeño académico, así como por cada estrategia y subescala. Posteriormente, se estimó la correlación entre el desempeño académico y las estrategias de las competencias cognitivas, considerando como niveles de correlación: débil en intervalo de $0-0,25$, media de $0,26-0,50$, considerable de 0,51-0,75 y fuerte $0,76-1$.
La segunda parte consideró la prueba ANOVA para realizar una comparación de la media entre subescalas, utilizando como factor el género, la edad, y el grado de escolaridad del padre y la madre. La prueba ANOVA se refiere al análisis de varianza de un factor para estudiar el efecto de una o más variables independientes sobre una variable de respuesta. Esta prueba estadística permite analizar si los grupos difieren significativamente entre sí en cuanto a sus medias y varianzas (Hernández et al., 2010).

\section{Resultados}

Una descripción demográfica de los participantes del estudio se presenta en la figura 1. Sus edades oscilan entre 17 y 25 años, un $63.9 \%$ eran femeninos, y un $36.1 \%$ masculino. 


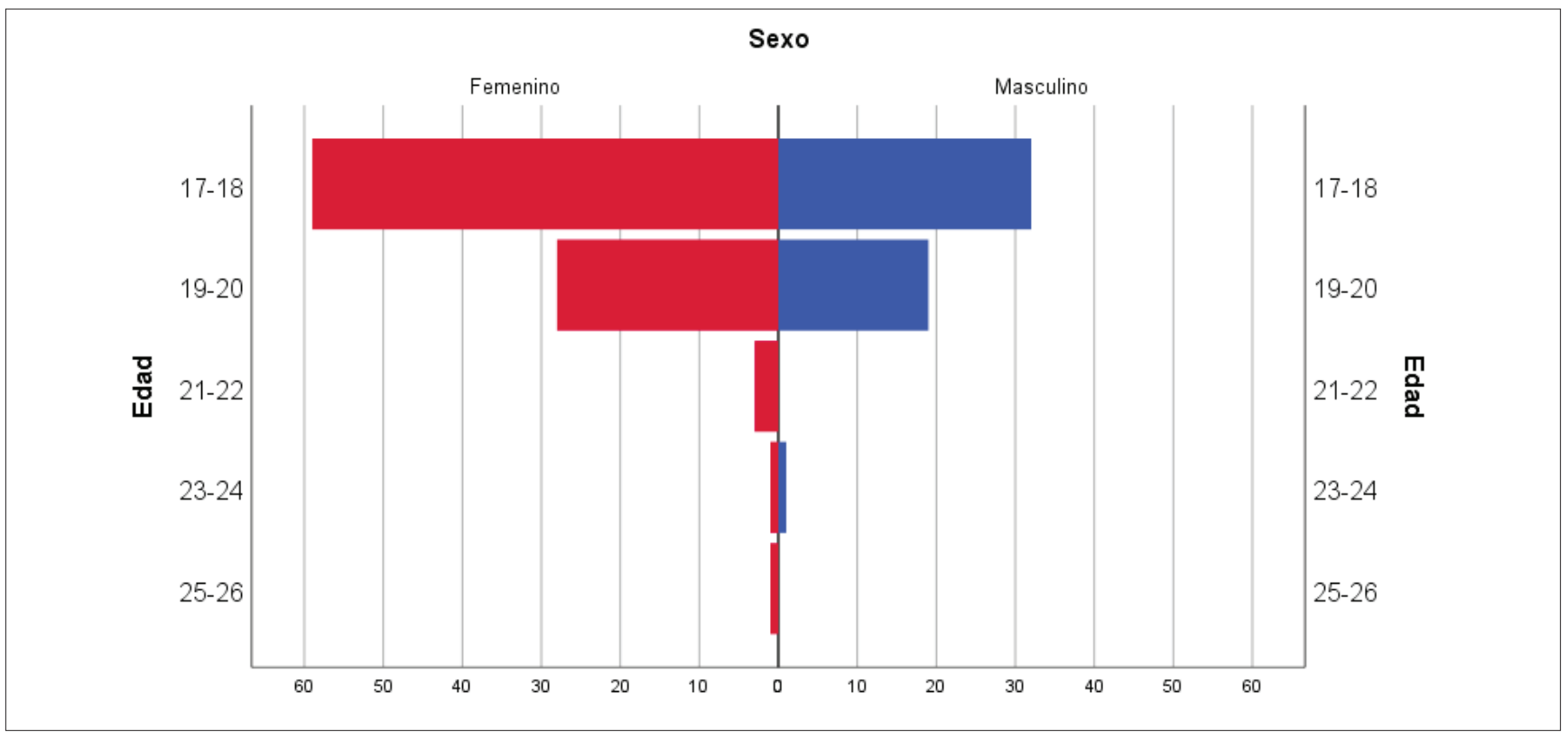

Figura 1: Pirámide poblacional de Sexo contra edad.

La media en el desempeño académico de la población fue de 9,22, en una escala del 1 al 10, con una desviación estándar de 0,41. La calificación más baja reportada por los participantes fue de 7,00 y la más alta de 10,00. Por tal razón se considera a la población como un grupo con rendimiento académico alto, lo cual es congruente con los parámetros de selección del programa de medicina. Al buscar una correlación entre el desempeño académico con la media en las diferentes estrategias, y subescalas de estrategias se encontraron correlaciones entre $-0,01$ y 0,16 consideradas como débil (tabla 2).

Tabla 2: Correlación entre el desempeño académico y las subescalas de las competencias cognitivas.

\begin{tabular}{|c|c|c|c|}
\hline Escala & Subescala & Correlación & Interpretación \\
\hline \multirow{4}{*}{$\begin{array}{l}\text { Estrategias } \\
\text { afectivas, } \\
\text { de apoyo y } \\
\text { control }\end{array}$} & $\begin{array}{l}\text { Estrategias motiva- } \\
\text { cionales. }\end{array}$ & 0,06 & Correlación débil \\
\hline & $\begin{array}{l}\text { Componentes } \\
\text { afectivos. }\end{array}$ & $-0,01$ & Correlación débil \\
\hline & $\begin{array}{l}\text { Estrategias meta- } \\
\text { cognitivas. }\end{array}$ & 0,03 & Correlación débil \\
\hline & $\begin{array}{l}\text { Estrategias de con- } \\
\text { trol del contexto, } \\
\text { interacción social y } \\
\text { manejo de recursos. }\end{array}$ & 0,16 & Correlación débil \\
\hline \multirow{2}{*}{$\begin{array}{l}\text { Estrategias } \\
\text { relacionadas } \\
\text { con el pro- } \\
\text { cesamiento } \\
\text { de la infor- } \\
\text { mación }\end{array}$} & $\begin{array}{l}\text { Estrategia de bús- } \\
\text { queda y selección } \\
\text { de información. }\end{array}$ & $-0,13$ & Correlación débil \\
\hline & $\begin{array}{l}\text { Estrategias de pro- } \\
\text { cesamiento y uso } \\
\text { de la información. }\end{array}$ & 0,03 & Correlación débil \\
\hline
\end{tabular}

Al analizar la media que los participantes con diferentes niveles de desempeño académico obtuvieron en las diferentes subescalas, se encuentra que los estudiantes con menor desempeño académico utilizan más las estrategias motivacionales $(4,25)$, que aquellos con mejor desempeño académico $(3,92)$; sin embargo, esta diferencia no es estadísticamente significativa (valor de $p=0,348$ ). En las subescalas de componentes físicos, estrategias metacognitivas y estrategias de procesamiento y uso de información no se observó una tendencia clara, y no se encontraron diferencias estadísticamente significativas. En la estrategia de control de contexto parece que los estudiantes con mejor desempeño académico tienen una media superior en esta subescala; sin embargo, esta diferencia tampoco es estadísticamente significativa (valor de $p=0,257$ ). Los estudiantes con menor desempeño académico utilizan más las estrategias de búsqueda y selección de información $(4,25)$, que aquellos con mejor desempeño académico $(3,80)$; sin embargo, esta diferencia no es estadísticamente significativa (valor de $\mathrm{p}=$ 0,478 ). Estos resultados se presentan en la tabla 3.

La estadística descriptiva encontró las estrategias de aprendizaje con medias más altas fueron motivación intrínseca con una media de 4,69 y desviación estándar de 0,56 y valor de la tarea con una media de 4,67 y desviación estándar de 0,54 Las tendencias menos favorables fueron en atribuciones externas con media de 2,55 y una desviación estándar de 0,78 y repetición simple con una media de 2,82 y una desviación de 1,12. Estos resultados se presentan en la tabla 4. 
López \& Heredia.

Tabla 3: Media por subescala de estrategias de acuerdo con el desempeño académico de los participantes.

\begin{tabular}{|c|c|c|c|c|c|c|c|c|}
\hline \multirow[b]{2}{*}{ Escala } & \multirow[b]{2}{*}{ Subescala } & \multicolumn{7}{|c|}{ Desempeño académico } \\
\hline & & 7,0 & 8,0 & 8,5 & 9,0 & 9,5 & 10,0 & $p$-value \\
\hline \multirow{4}{*}{$\begin{array}{l}\text { Estrategias afectivas, } \\
\text { de apoyo y } \\
\text { control }\end{array}$} & Estrategias motivacionales. & 4,25 & 3,63 & 3,63 & 3,79 & 3,79 & 3,92 & 0,348 \\
\hline & Componentes físicos. & 3,37 & 3,00 & 3,50 & 3,62 & 3,52 & 3,53 & 0,576 \\
\hline & Estrategias metacognitivas. & 4,38 & 3,77 & 4,08 & 4,09 & 4,05 & 4,31 & 0,751 \\
\hline & Estrategias de control del contexto, interacción social y manejo de recursos. & 3,75 & 4,25 & 3,62 & 4,13 & 4,19 & 4,32 & 0,257 \\
\hline \multirow{2}{*}{$\begin{array}{l}\text { Estrategias relacionadas } \\
\text { con el procesamiento } \\
\text { de la información }\end{array}$} & Estrategia de búsqueda y selección de información. & 4,25 & 4,75 & 4,18 & 3,96 & 3,91 & 3,80 & 0,600 \\
\hline & Estrategias de procesamiento y uso de la información. & 4,31 & 4,75 & 3,81 & 4,04 & 4,03 & 4,18 & 0,478 \\
\hline
\end{tabular}

*Diferencia estadísticamente significativa

Tabla 4: Detalle de tendencia por estrategia.

\begin{tabular}{|c|c|c|c|c|}
\hline Escala & Subescala & Estrategia & Media & Desviación estándar \\
\hline \multirow{15}{*}{$\begin{array}{l}\text { Estrategias afectivas, } \\
\text { de apoyo y control } \\
\text { (automanejo) }\end{array}$} & \multirow{8}{*}{ Estrategias motivacionales. } & Atribuciones externas. & 2,55 & 0,78 \\
\hline & & Atribuciones internas. & 3,57 & 0,54 \\
\hline & & Autoevaluación. & 4,13 & 0,63 \\
\hline & & Autoeficacia y expectativas & 4,58 & 0,49 \\
\hline & & Concepción de la inteligencia como modificable. & 3,33 & 0,62 \\
\hline & & Motivación extrínseca. & 2,85 & 0,85 \\
\hline & & Motivación intrínseca. & 4,69 & 0,56 \\
\hline & & Valor de la tarea. & 4,67 & 0,54 \\
\hline & \multirow{2}{*}{ Componentes físicos. } & Estado físico y anímico. & 3,99 & 0,76 \\
\hline & & Ansiedad. & 3,14 & 0,58 \\
\hline & \multirow{3}{*}{ Estrategias metacognitivas. } & Conocimiento de objetivos y criterios de evaluación. & 4,25 & 0,67 \\
\hline & & Planificación. & 3,68 & 0,80 \\
\hline & & Evaluación, control, autorregulación. & 4,36 & 0,49 \\
\hline & \multirow{2}{*}{$\begin{array}{l}\text { Estrategias de control del } \\
\text { contexto, interacción social } \\
\text { y manejo de recursos. }\end{array}$} & Control de contexto. & 4,19 & 0,72 \\
\hline & & $\begin{array}{l}\text { Habilidades de interacción social y aprendizaje con } \\
\text { compañeros. }\end{array}$ & 4,10 & 0,79 \\
\hline \multirow{10}{*}{$\begin{array}{l}\text { Estrategias relacio- } \\
\text { nadas con el proce- } \\
\text { samiento de la infor- } \\
\text { mación }\end{array}$} & \multirow{2}{*}{$\begin{array}{l}\text { Estrategias de búsqueda y } \\
\text { selección de información. }\end{array}$} & Conocimiento de fuentes y búsqueda de información. & 3,88 & 0,82 \\
\hline & & Selección de información. & 4,03 & 0,65 \\
\hline & \multirow{8}{*}{$\begin{array}{l}\text { Estrategias de procesamien- } \\
\text { to y uso de la información. }\end{array}$} & Adquisición de información. & 4,23 & 0,81 \\
\hline & & Elaboración de información. & 4,40 & 0,71 \\
\hline & & Organización de información. & 3,98 & 0,95 \\
\hline & & Personalización y creatividad, pensamiento crítico. & 4,19 & 0,72 \\
\hline & & Almacenamiento. Nemotécnica. & 4,22 & 0,86 \\
\hline & & Transferencia y uso de información. & 4,51 & 0,57 \\
\hline & & Manejo de recursos para utilizar la información. & 3,98 & 0,74 \\
\hline & & Almacenamiento. Simple repetición. & 2,82 & 1,12 \\
\hline
\end{tabular}


En segundo lugar, se consideró la prueba ANOVA para comparar la media entre subescalas, utilizando como factor el género, la edad, y el grado de escolaridad del padre y la madre. La tabla 5 indica la media en cada una de las subescalas estratificando por género donde la única diferencia significativa se encuentra en la subescala de estrategia de búsqueda y selección de información. El análisis de varianza considerando como factor la edad, escolaridad del padre y de la madre, no reportó diferencias significativas.

Tabla 5: Media por subescala de estrategias de acuerdo con el género de los participantes.

\begin{tabular}{|c|c|c|c|c|}
\hline Escala & Subescala. & Femenino & Masculino & Valor de $p$ \\
\hline \multirow{4}{*}{$\begin{array}{l}\text { Estrategias afectivas, } \\
\text { de apoyo y } \\
\text { control }\end{array}$} & Estrategias motivacionales. & 3,79 & 3,79 & 0,89 \\
\hline & Componentes físicos. & 3,54 & 3,59 & 0,48 \\
\hline & Estrategias metacognitivas & 4,08 & 4,10 & 0,77 \\
\hline & $\begin{array}{l}\text { Estrategias de control del } \\
\text { contexto, interacción social } \\
\text { y manejo de recursos. }\end{array}$ & 4,12 & 4,17 & 0,65 \\
\hline \multirow{2}{*}{$\begin{array}{l}\text { Estrategias relacionadas con el procesamiento } \\
\text { de la información }\end{array}$} & $\begin{array}{l}\text { Estrategia de búsqueda y } \\
\text { selección de información. }\end{array}$ & 3,86 & 4,11 & $0,02^{*}$ \\
\hline & $\begin{array}{l}\text { Estrategias de procesamiento } \\
\text { y uso de la información. }\end{array}$ & 4,04 & 4,03 & 0,86 \\
\hline
\end{tabular}

*Diferencia estadísticamente significativa

\section{Discusión}

Los resultados del desempeño académico son congruentes con los parámetros de selección del programa de medicina, los cuales son superiores a los criterios de entrada a otros programas de licenciatura. Se cuenta con estudiantes con un alto perfil académico, quienes comparten un deseo por aprender (Garbanzo, 2007) y lo traducen a resultados positivos en las métricas de evaluación del aprendizaje, como exámenes, proyectos o calificaciones del curso (Edel, 2003).

Este trabajo tiene un impacto potencialmente importante para el campo de la educación médica, porque permite acompañar a los estudiantes en el desarrollo de estrategias más efectivas para su aprendizaje. Esto se vuelve muy relevante especialmente con estudiantes de nuevo ingreso a una carrera profesional, que enfrentan ya de por sí un reto importante de acoplarse a un modelo educativo más autodirigido en el que deben demostrar mayor responsabilidad por su aprendizaje.

Los altos resultados obtenidos en las estrategias de control del contexto son similares a los resultados de Navea que obtuvo en sus participantes una alta percepción de importancia en las estrategias de gestión del tiempo y del lugar del estudio, y las estrategias de aprendizaje con otros compañeros y búsqueda de autoayuda (Navea, 2018). Sin embargo, los resultados de estrategias metacognitivas son contrarios a este estudio, ya que en el estudio de Navea esta estrategia obtuvo los valores más bajos.
El estudio de las estrategias utilizadas por los participantes permite comprender la vinculación entre sus recursos para controlar la movilización y el desarrollo de su aprendizaje. Contrario a lo reportado por otros estudios, no se encontró una relación directa con sus estrategias relacionadas con el procesamiento de información (Valdez et al., 2017). Los estudiantes de medicina destacan en la activación a través de su motivación intrínseca, lo que Marzano \& Kendall, (2007) describen como sistema interno, son estudiantes autorregulados que no requieren un control externo para decidir involucrarse en una tarea.

El estudio tiene algunas limitaciones, como el hecho de que fue realizado en una sola institución educativa por lo que podría no representar otros contextos inclusive en el mismo país. También se consideró solamente el programa de medicina, y las estrategias aplicadas podrían ser distintas aun dentro del área de salud. En este análisis se integró a estudiantes que participaron voluntariamente en el estudio, una interrogante es si eso no introduce un sesgo ya que estos estudiantes de por si una población diferente como más ordenados y responsables, ya que al participar como voluntarios en un estudio indicaría que comprometen tiempo a dedicar, y que no sea representativa del resto de los estudiantes de medicina. Otra limitación tiene que ver con el momento académico en el cual se aplicó la encuesta, sin duda la pandemia ha tenido un impacto en las estrategias de aprendizaje al depender ahora de un modelo educativo a distancia. Los estudiantes han debido regular sus emociones e integrar nuevos recursos que favorezcan 
su aprendizaje. Además de señalar que el desempeño académico por ser un fenómeno multideterminado es mucho más complejo que limitar su comprensión a algunas variables, pero que pueden aportar evidencia para enfocar mejor, en este caso, el apoyo para que los estudiantes de medicina aprendan y utilicen estrategias de aprendizaje más complejas para su propio beneficio.

\section{Conclusión}

El estudio de las estrategias de aprendizaje que utilizan los estudiantes presenta una ventana para entender cuáles de estas favorecen un buen desempeño en los programas, además de proponer adecuaciones curriculares que enfaticen aquellas necesarias para convertirse en aprendices a lo largo de la vida. De acuerdo con el objetivo del estudio planteado, se refuta que exista una relación fuerte entre las estrategias de aprendizaje y el desempeño académico reportado por estudiantes de medicina. Las estrategias afectivas, en particular las motivaciones que tuvieron las tendencias más favorables, como la motivación intrínseca y el valor de la tarea, pudieran ser determinantes para que los estudiantes se adapten a entornos cambiantes y desafiantes como aquellos que se han enfrentado en la reciente crisis sanitaria.

Futuros estudios deberían abordar ese tema y podrían ahondar en el origen de la práctica de estas estrategias, también sería conveniente integrar otros países de Latinoamérica que comparten algunos elementos culturales y retos en el contexto educativo, y contrastar las diferencias entre las correlaciones observadas.

Como aportación al campo de estudio, esta investigación deja en evidencia que estrategias que antes eran la base de estudiar una carrera en salud, como el almacenamiento y la simple repetición de contenido han ido disminuyendo, dando lugar a la activación de niveles cognitivos más altos, como el metacognitivo en el que el individuo propone metas y genera estrategias que le permitan alcanzar las metas.

\section{Contribuciones y reconocimientos}

Las autoras declaran no tener fuentes de financiamiento. Mildred López realizó la investigación en campo, análisis, escritura del primer manuscrito, administración del proyecto, revisión y edición del manuscrito final. Mientras que Yolanda Heredia Escorza efectúo conceptualización, metodología, revisión y edición del manuscrito final. Las autoras declaran no tener ningún conflicto de interés.

\section{Referencias}

Biwer F, Egbrink MGA, Aalten P. \& Bruin ABH. (2020). Fostering Effective Learning Strategies in Higher Education - A Mixed - Methods Study. Journal of Applied Research in Memory and Cognition 9, 186-203.
Edel R. (2003). El rendimiento académico: concepto, investigación y desarrollo. Revista Electrónica Iberoamericana sobre Calidad, Eficacia y Cambio en Educación 1. Accedido en: https://www.redalyc.org/ articulo.oa?id=55110208 el 27 de marzo de 2021.

Gagné RM. (1996). Learning processes and instruction. Training Research Journal 1, 17-28.

Garbanzo GM. (2007). Factores asociados al rendimiento académico en estudiantes universitarios, una reflexión desde la calidad de la educación superior pública. Revista Educación 31, 43-63.

Gargallo B, Suárez-Rodriguez JM. \& Pérez-Perez C. (2009). El cuestionario CEVEAPEU. Un instrumento para la evaluación de las estrategias de aprendizaje de los estudiantes universitarios. RELIEVE 15, 1- 31.

Hayat AA, Shateri K, Amini M, \& Shokrpour N. (2020). Relationships between academic self-efficacy, learning-related emotions, and metacognitive learning strategies with academic performance in medical students: a structural equation model. BMC Medical Education 20, 76.

Hernández R, Fernández C. \& Baptista MP. (2010). Metodología de la investigación. McGraw Hill, México.

Marzano R, \& Kendall J. (2007). The new taxonomy of educational objectives. Corwin Press, EEUU.

Navea A. (2018). El aprendizaje autorregulado en estudiantes de ciencias de la salud: recomendaciones de mejora de la práctica educativa. Educación Médica 19, 193-200.

Obergriesser S. \& Stoeger H. (2020). Student's emotions of enjoyment and boredom and their use of cognitive learning strategies - How do they affect one another? Learning and Instructions 66, 101285.

Páez I. (2006). Estrategias de aprendizaje -investigación documental- (parte A). Laurus 12, 254-266.

Pérez V. \& La Cruz A. (2014). Estrategias de enseñanza y aprendizaje de la lectura y escritura en educación primaria. Zona Próxima 21, 1-16.

Pintrich P. \& García T. (1993). Intraindividual differences in students' motivation and self-regulated learning. German Journal of Educational Psychology 7, 99-107.

Pintrich P, Smith T, García W. \& McKeachie A. (1991). A manual for the use of the Motivated Strategies for Learning Questionnaire (MSLQ). National Center for Research to Improve Postsecondary Teaching and Learning University of Michigan, Michigan. 
López \& Heredia.

Quiroga-Garza ME, Flores-Marín DL, Cantú-Hernández RR, Eraña I. \& Lopez M. (2020). Effects of a vocational program on professional orientation. Heliyon 6, e03860.

Ramos GRM. \& Pacheco MEP. (2017). Factores incidentes en el rendimiento académico estudiantil de Ingeniería en Sistemas de Información de la FAREM-Matagalpa, 2012-2016. Revista Científica FAREM-Estelí 24, 5-26.

Rinaudo MC, Chiecher A. \& Donolo D. (2003). Motivación y uso de estrategias en estudiantes universitarios. Su evaluación a partir del Motivated Strategies Learning Questionnaire. Anales de psicología 19, 107-119.
Salazar I. \& Heredia Y. (2019). Estrategias de aprendizaje y desempeño académico en estudiantes de Medicina. Educación Médica 20, 256-262.

Schunk D. (1997). Teorías del Aprendizaje. Prentice Hall, México. Valdez García JE, López Cabrera MV. \& Olivares Olivares SL. (2017). Definición de metas de aprendizaje en estudiantes de pregrado en un curso de Historia de la Medicina. Educación Médica 19, 105-110. 